Original Research

\title{
Physical Activity Behaviors and COVID-19 Symptoms
}

\author{
Zachary Zeigler *, Jacob Lawrence, Alexa Jamison, Pete Salzano
}

The College of Science Engineering and Technology, Grand Canyon University, Phoenix, AZ, USA; EMails: zachary.zeigler@gcu.edu; Jacob.Lawrence@gcu.edu; $\quad$ Alexa.Jamison@gcu.edu; Pete.Salzano@gcu.edu

* Correspondence: Zachary Zeigler; E-Mail: zachary.zeigler@gcu.edu

Academic Editor: Sok Cheon Pak and Soo Liang Ooi

Special Issue: Complementary, Traditional, and Integrative Medicine for COVID-19

OBM Integrative and Complementary Medicine

2021, volume 6 , issue 3

doi:10.21926/obm.icm.2103027
Received: August 02, 2021

Accepted: August 30, 2021

Published: September 02, 2021

\begin{abstract}
Statistical modeling indicated that COVID-19 would have afflicted more than $60 \%$ of the US population. Social distancing, self-quarantine, and widespread shutdowns were imposed. The push to stay at home and the decreased availability of exercise facilities have potentially reduced physical activity (PA). The purpose of this observational, correlational study was to determine if there is a relationship between PA level and symptoms of COVID-19. Subjects were asked to complete a single Survey Monkey questionnaire. The survey asked demographic questions, PA behaviors, and questions relating to COVID-19 symptoms. Chisquare analysis was run to determine frequency differences within the PA group and specific COVID-19 symptoms. Hierarchical regression analysis was run to determine if PA was a unique predictor of the number COVID-19 symptoms experienced. Sixty male and 85 female $(n=145)$ subjects completed this study. Subjects were $25.6 \pm 11.2 \mathrm{yr}$ old with an average BMI of $25.3 \pm$ $5.2 \mathrm{~kg} / \mathrm{m}^{2}$. There were no statistically significant differences between those who achieved PA guidelines compared to those who did not on any single symptom besides fever. A greater number of participants who did not achieve PA guidelines reported no fever (69.2\% vs. $46.6 \%$, $p=0.018$ ). Age, gender, ethnicity, and BMI did not significantly predict the number COVID-19 symptom $(p=0.312)$. Only the addition of moderate MET-minutes week $^{-1}$ made the full model
\end{abstract}

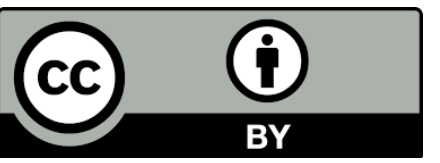

(C) 2021 by the author. This is an open access article distributed under the conditions of the Creative Commons by Attribution License, which permits unrestricted use, distribution, and reproduction in any medium or format, provided the original work is correctly cited. 
statistically significant $\left(p=0.009, R^{2}=.093\right)$. Moderate MET-minutes. week $^{-1}$ was the only independent predictor of COVID-19 symptoms ( $p=0.009$ ). In conclusion, increased levels of moderate-intensity PA may protect against acquiring a greater number of COVID-19 symptoms.

\section{Keywords}

COVID-19; physical activity; exercise; lifestyle

\section{Introduction}

On January $30^{\text {th }}, 2020$, the WHO declared the severe acute respiratory syndrome coronavirus 2 (SARS-CoV-2), the virus that causes the novel coronavirus disease 2019 (COVID-19), pandemic a global health emergency [1]. Statistical modeling indicated that without mitigation, COVID-19 would have afflicted more than $60 \%$ of the US population [2]. The leading damage of the virus is to the respiratory system, immune system, and exacerbating underlying medical conditions [3]. Several health organizations such as the US Centers for Disease Control and Prevention [4] and the WHO [1] issued safety recommendations to reduce exposure to and transmission of the virus. Social distancing, self-quarantine, and widespread shutdowns were imposed to flatten the epidemic curve. The objective of these measures was to curb the effects of the virus on the healthcare system, morbidity, and mortality [5].

Because of these precautions, most exercise and recreational facilities were forced to close or alter routine operating procedures. Closing exercise facilities is concerning; most modern countries were already experiencing a pandemic of physical inactivity [6]. The push to stay at home and the decreased availability of exercise facilities have potentially reduced physical activity (PA) further [7]. For example, a cross-sectional study of 37,252 participants from France found that $63 \%$ reported increased sedentary time, with an average of seven hours per day spent sitting during lockdown [8]. In addition, reports show an overall decrease in walking during COVID-19 self-quarantine [9]. A worldwide sample of roughly 450,000 subscribers of a popular fitness app found that daily step counts were reduced by roughly $30 \%$ within 30 days of pandemic declaration [10]. Countries with more strict mandates, such as Italy, showed a $49 \%$ reduction in step counts [10].

A dramatic reduction in PA is worrisome as prior literature has shown that just two days of inactivity are associated with muscle wasting, alterations in glucose homeostasis, and insulin sensitivity reductions [11]. Also, increased sedentary time was highlighted as a primary cause of the increased back pain that has been reported during the pandemic [12]. Physical inactivity is directly [13] and indirectly related to inadequate immune response through its effects on obesity development [14]. On the other hand, daily exercise may help combat COVID-19 by boosting the immune system and counteracting comorbidities such as obesity, diabetes, hypertension, and heart conditions that increase severe COVID-19 illness [15]. Animal models suggest that pre-infection exercise training at moderate intensities improves morbidity and mortality to viral infections [1618]. However, some data suggest that high-intensity exercise may lead to poorer outcomes in response to respiratory viral infections [19-21]. Thus, a J-shaped theorized relationship between exercise intensity and respiratory illness has emerged [22]. 
Exercise-induced adaptations enhance the effectiveness of the immune system [23]. Additionally, although not always consistent [22], exercise-induced changes to the immune system may also affect respiratory virus susceptibility [24]. An extensive study by Hamar et al. found that a healthy lifestyle that includes PA was protective against getting COVID-19 [25]. However, little is known about how PA interacts with the immune system to impact specific COVID-19 symptoms once the virus is acquired. A recent paper looking at lifestyle factors and COVID-19 disease severity in Iranian subjects found that those with lower levels of PA were affected more severely by COVID19 [26]. Because the researchers used CT scan findings and positive PCR tests for inclusion criteria, mild cases were not included in the study. Emerging data has also shown that participants with lower exercise capacity had an increased risk of becoming hospitalized from COVID-19 [27]. The health impacts of high PA levels are not identical to the health impacts of a high measured cardiovascular fitness level [28]. Thus, there are still open questions regarding the role of PA on specific COVID-19 symptoms [29].

Eighty percent of confirmed COVID-19 cases show little or even no disease symptoms [30, 31]. Whether or not those who are more physically active express fewer symptoms is unknown. Hence, the purpose of this observational, correlational study is to determine if there is a relationship between PA level and the expression of COVID-19 symptoms.

\section{Methods}

\subsection{Subjects}

\subsubsection{Population}

A priori G*Power using bivariate correlational analysis was used as the research design to approximate sample size. An estimated effect size of .3, an alpha error of 0.05 , and a power of .95 projected that 111 subjects were needed to power this study adequately. Subjects were included if they were over the age of $18 \mathrm{yr}$ and self-reported that they have had a confirmed positive test for COVID-19. Potential subjects were asked, "have you received a positive COVID-19 test?" Subjects were excluded if they are current or former smokers and have known pulmonary, cardiovascular, renal, metabolic diseases, or cancer. A research announcement was sent out via social media to possible participants. Snowball sampling was also used such that each participant was asked who they knew who has tested positive that may like to participate.

\subsection{Material and Methods}

\subsubsection{Study Design}

The research design used was a quantitative correlational design. The Institutional Review Board at the host institution approved this study. Once the subjects agreed to participate in the study, they received a link to a Survey Monkey questionnaire. Informed consent was at the beginning of the online survey. Subjects were asked to complete a single Survey Monkey questionnaire. The survey asked demographic questions, PA behaviors, and questions relating to the severity of COVID19 symptoms. 


\subsubsection{Demographics Questionnaire}

Descriptive information was collected to characterize the subject population. Subjects were asked to reveal their age, gender, ethnicity, height, and weight. These variables were essential to obtain as it has been shown that each impacts COVID-19 susceptibility and severity [3].

The International Physical Activity Questionnaire short form (IPAQ-SF) was chosen to measure PA behaviors. The IPAQ-SF has been found valid and reliable [32, 33]. According to official IPAQ-SF guidelines, data are summed within each item (i.e., vigorous-intensity, moderate-intensity, and walking) to estimate the total amount of time spent engaged in PA per week [32, 33]. Total weekly PA (MET-min. week $^{-1}$ ) was estimated by adding the products of reported time for each item by a MET value specific to each PA category. The MET values used were the original values (original IPAQ) based on the official IPAQ guidelines for young and middle-aged adults (18-65 yr): vigorous $\mathrm{PA}=$ 8.0 METs, moderate PA = 4.0 METs, and walking = 3.3 METs. Those who reported $\geq 150 \mathrm{~min} /$ week of moderate intensity PA exercise per week and, or $\geq 75 \mathrm{~min} /$ week of vigorous intensity PA, or an equivalent combination of both moderate- and vigorous intensity aerobic exercise were considered to meet PA guidelines [34]. Combination was calculated by adding moderate minutes per week plus two times vigorous minutes per week. Meeting moderate to vigorous intensity PA (MVPA) was determined if combination equated to $\geq 150$.

The primary investigator created the questionnaire used to assess COVID-19 symptoms. Questions were based on reported clinical characteristics of those diagnosed with COVID-19 [30]. Subjects were asked on an ordinal scale if they had a fever, persistent cough, unusual fatigue, headache, unusual shortness of breath, sore throat, loss of smell/taste, unusually hoarse voice, unusual chest pain or tightness, unusual abdominal pain, diarrhea, or nausea. Subjects were also asked to report if they were admitted to the hospital due to COVID-19. If admitted, subjects were asked what treatment they were given (oxygen and fluids, non-invasive ventilation, invasive ventilation). In addition to the ordinal questions asked, a COVID-19 symptom index was created to estimate how many symptoms were experienced. All positive symptoms were added together to create an interval symptom index. For example, assume a subject was asked, 'did you have a fever?' 1 = yes, 2 = no, and 'did you have a persistent cough?' 1 = yes, 2 = no. If the subject answered yes to both, the symptom index would be 2 . Thus, a lower score represents increased symptoms.

\subsubsection{Statistical Analysis}

All statistical analyses were performed using SPSS software version 27 (SPSS 27.0 IBM Corporation, Armonk, New York, USA). Data are expressed as means \pm standard deviation. Data were analyzed for normality, and values with skewed or kurtotic distributions were transformed to achieve normality. Descriptive statistics were used for the demographics of the participants. Subjects were dichotomized into two groups; those who achieved the public health PA guidelines of 150 minutes a week of MVPA and those who did not. Chi-square analysis was run to determine frequency differences within the PA group and specific COVID-19 symptoms. Hierarchical regression analysis was run to determine if PA was a unique predictor of COVID-19 symptoms. All $p$-values were calculated assuming a two-tailed hypothesis, and $p<.05$ was considered statistically significant. 


\section{Results}

Sixty male and 85 female $(n=145)$ subjects completed this study. Subjects were $25.6 \pm 11.2 \mathrm{yr}$ old with an average BMI of $25.3 \pm 5.2 \mathrm{~kg} / \mathrm{m}^{2} .57 \%$ of subjects were Caucasian, $32 \%$ were Hispanic, $6 \%$ were African American, and 5\% were "other". Table 1 details the number of minutes per week subjects participated in the different PA categories. PA values are like what has been reported in other research during the COVID-19 pandemic within a similar population [35].

Table 1 Physical Activity Behaviors.

\begin{tabular}{cc}
\hline Physical activity category & Mean \pm SD \\
\hline Minutes spent walking & $244.8 \pm 250.4$ \\
Walking MET values & $1013.2 \pm 1734.1$ \\
Minutes spent in moderate activity & $138.3 \pm 169.8$ \\
Moderate MET values & $652.9 \pm 852.6$ \\
Minutes spent in vigorous activity & $178 \pm 181.9$ \\
Vigorous MET values & $1708.5 \pm 1919.3$ \\
Total weekly activity (min) & $565.3 \pm 447.9$ \\
Total MET values & $3130.8 \pm 28.10 .5$ \\
Sitting (min/day) & $384.1 \pm 264.4$ \\
\hline
\end{tabular}

The most reported symptoms of COVID-19 were unusual fatigue (91\%), headaches (79\%), shortness of breath (75\%), and loss of smell or taste (72\%) (for a complete list of symptoms, see Table 2).

Table 2 COVID-19 Symptoms.

\begin{tabular}{cc}
\hline Symptom & Percentage of participants experiencing symptom (N) \\
\hline Fever & $47 \%(68)$ \\
Persistent cough & $35 \%(49)$ \\
Unusual fatigue & $91 \%(131)$ \\
Headache & $79 \%(115)$ \\
Shortness of breath & $55 \%(79)$ \\
Sore throat & $59 \%(85)$ \\
Loss of smell or taste & $72 \%(104)$ \\
Hoarse voice & $23 \%(33)$ \\
Chest pain & $44 \%(64)$ \\
Abdominal pain & $21 \%(30)$ \\
Diarrhea & $32 \%(47)$ \\
\hline
\end{tabular}




\begin{tabular}{cc}
\hline Nausea & $35 \%(50)$ \\
Admitted to hospital & $3 \%(5)$ \\
\hline
\end{tabular}

$N=145$

One hundred and six subjects achieved the public health guidelines of $150 \mathrm{~min} /$ week of MVPA while 39 subjects did not. Although not statistically significant, compared to those who did not achieve PA guidelines, participants who reported they did achieve PA guidelines had more people report a lack of fatigue, headache, shortness of breath, hoarse voice, abdominal pain, diarrhea, and nausea (Table 3). However, there were significantly more people reporting no fever within those who did not achieve PA guidelines than those who did (69.2\% vs. $46.6 \%, p=0.018$ ).

Table 3 Percentage of Participants Not Experiencing the Symptom.

\begin{tabular}{cccc}
\hline Symptom & Inactive & Active & P-Value \\
\hline Fever & $69.2 \%$ & $46.6 \%$ & 0.018 \\
Persistent Cough & $67.6 \%$ & $58.8 \%$ & 0.362 \\
Unusual Fatigue & $7.7 \%$ & $12.5 \%$ & 0.142 \\
Headache & $10.3 \%$ & $20.5 \%$ & 0.161 \\
Shortness of Breath & $34.2 \%$ & $50.0 \%$ & 0.270 \\
Sore Throat & $41.0 \%$ & $47.7 \%$ & 0.484 \\
Loss of Taste or Smell & $25.6 \%$ & $25.0 \%$ & 0.939 \\
Hoarse Voice & $71.8 \%$ & $83.0 \%$ & 0.151 \\
Chest Pain & $59.0 \%$ & $53.4 \%$ & 0.561 \\
Abdominal Pain & $74.4 \%$ & $85.2 \%$ & 0.142 \\
Diarrhea & $59.0 \%$ & $70.5 \%$ & 0.205 \\
Nausea & $53.8 \%$ & $70.5 \%$ & 0.070 \\
Admitted to Hospital & $97.4 \%$ & $96.6 \%$ & 0.801 \\
\hline
\end{tabular}

Hierarchical regression was run with age, gender, BMI, and ethnicity in the first block and the different PA measurements in the second block. The COVID-19 symptom index was the criterion variable. Age, gender, ethnicity, and BMI did not significantly predict COVID-19 symptom severity $(p=0.312)$. The addition of vigorous MET-minutes $\cdot w^{-1}{ }^{-1}$ in the second block did not significantly predict COVID-19 symptoms ( $p=0.332$ ). Vigorous MET-minutes-week ${ }^{-1}$ was then replaced with Moderate MET-minutes $\cdot$ week $^{-1}$. The addition of moderate MET-minutes $\cdot$ week $^{-1}$ made the full model statistically significant $\left(p=0.009, R^{2}=.093\right.$ ). Moderate MET-minutes $\cdot$ week $^{-1}$ was the only independent predictor of COVID-19 symptoms $(p=0.009)$. Moderate MET-minutes $\cdot$ week $^{-1}$ was then 
replaced with walking MET-minutes week $^{-1}$. The full model was not statistically significant $(p=$ 0.719). Walking MET-minutes week $^{-1}$ was replaced with total MET-minutes $\cdot$ week $^{-1}$. The full model was not statistically significant $(p=0.298)$. Total MET-minutes $\cdot$ week $^{-1}$ was replaced with sitting time. The full model was not statistically significant $(p=0.296)$.

\section{Discussion}

The current study included 145 participants who reported having tested positive to COVID-19 to determine the relationship between PA and COVID-19 symptoms. The main finding from this study was that moderate-intensity PA may decrease COVID-19 symptoms, and this was not seen with higher intensity PA levels. Our findings align with past work that reports that PA and respiratory tract infection mainly correlate with exercise intensity [36, 37].

Our work also aligns with emerging data concerning the COVID-19 virus and exercise. A recent large cross-sectional study concluded that regular sports participation might decrease disease severity. Indeed, these authors found that hospitalizations of athletes who regularly participated in sports were 33\% lower than the nonathletic groups [38]. Additionally, Tavakoi looked crosssectionally at 206 participants who tested positive for COVID-19 to determine predictors of disease severity [26]. These researchers found that moderate-intensity PA, not vigorous, decreased COVID19 disease severity. Lastly, in a study of 48,440 subjects, it was found that those who reported to consistently meet PA guidelines were less likely to become hospitalized, admitted to the ICU, or die from COVID-19 complications [39].

Several physiological processes have been suggested to explain the relationship between PA and improved immune and lung function. For example, enhanced natural killer cell activity has been reported following moderate PA $[40,41]$. Improved immune function from PA can reduce the influx of inflammatory cells into the lungs, decrease pathogen load, improve disease outcomes, and decrease pro-inflammatory cytokines in the lungs or bronchoalveolar fluid $[16,18]$. Animal models confirm that the correlation between exercise intensity and influenza shows increased susceptibility with intense exercise [20] and decreased susceptibility with moderate exercise [17]. This may explain why the current study did not find a relationship between time spent in vigorous-intensity exercise or total PA on COVID-19 symptoms.

Age and gender were not independent predictors of COVID-19 severity in our study. In a multivariable analysis predicting hospitalization from COVID-19, it was found that the strongest predictor was an age of 75 years or older [42]. Hence, it was not surprising that the current study did not show age as a predictor when considering our mean age was 25 years old. Additionally, whether or not gender predicts COVID-19 disease severity is inconclusive, with some data showing the male gender predicts greater COVID-19 severity [42] while other data shows that women suffered longer durations of symptoms than men [26].

Interestingly, our study did not find BMI to predict COVID-19 symptoms. The majority of research points to increased disease severity with increased levels of BMI [42-44]. For instance, when predicting hospitalizations in New York from COVID-19, the strongest predictor was age, followed by obesity [42]. Additional research found that COVID-19 disease severity was associated with increased BMI categories [44]. However, Tavakol looked at risk factors of COVID-19 severity and found that obesity was not among the predictors [26]. Additionally, Sallis et al. found that it was not until BMI's got above $40 \mathrm{~kg} / \mathrm{m}^{2}$ that risk for hospitalization, ICU admittance, and death was 
increased [39]. The current study had an average BMI of $25.3 \pm 5.2 \mathrm{~kg} / \mathrm{m}^{2}$. Consequently, differences in findings may be due to a smaller number of participants in higher BMI categories within the current study. Differences between studies may also be explained by the various ways COVID-19 disease outcomes are being determined. The current study, for example, added up the number of COVID-19 symptoms. Lastly, the current study included those with much milder cases of COVID-19.

There are strengths to this study. First, prior research used CT scan findings and positive PCR tests for inclusion criteria [26]. Thus, mild cases were not included. Additionally, data on moderate PA in the general population are limited [45]. The current study provides information about the impact of moderate-intensity PA on symptoms of those who have mild cases of COVID-19.

The study has several weaknesses. Because of the sampling procedure, the participant population may be biased and not representative of the general population. Indeed, our population was relatively young, predominately Caucasian, and active. Additionally, all data is self-reported. Self-reported data can lead to incorrect reporting. This may be the reason that such a high percentage of the sample reported meeting PA guidelines. Lastly, our subjects all had relatively mild cases of COVID-19, as shown by the small number of hospitalizations $(n=4)$. Thus, our study may not adequately describe PA's ability to protect against severe cases. Our study would be most generalizable to young, fit, college-aged populations. Other populations should interpret these results with caution.

In conclusion, this study adds support that increased levels of moderate-intensity PA may protect against acquiring a greater number of COVID-19 symptoms. This is valuable information, quality of life during the COVID-19 pandemic has been reported to decrease [46], and many people may feel helpless to change their circumstances. Increasing levels of moderate-intensity PA is one area that the population could take control over that may decrease population disease burden.

\section{Author Contributions}

ZSZ provided study design, helped with data collection, data analysis, and manuscript preparation. AJ aided in data collection and manuscript preparation. JL provided data collection and manuscript preparation. PS aided in data collection and manuscript preparation.

\section{Funding}

There was no funding source for this study.

\section{Competing Interests}

There are no conflicts of interest to declare.

\section{References}

1. Velavan TP, Meyer CG. The COVID-19 epidemic. Trop Med Int Health. 2020; 25: 278-280.

2. Fink S. Worst-case estimates for US coronavirus deaths [Internet]. New York: The New York Times Company; 2020. Available from: http://www.mifami.org/Math110/ch10-The\%20WorstCase\%20Estimate\%20for\%20U.S.\%20Coronavirus\%20Deaths\%20-\%20The\%20New\%20York\%2 OTimes.pdf.

3. Mousavizadeh L, Ghasemi S. Genotype and phenotype of COVID-19: Their roles in pathogenesis. 
J Microbiol Immunol Infect. 2021; 54: 159-163.

4. Mclntosh K, Hirsch MS, Bloom A. Coronavirus disease 2019 (COVID-19) [Internet]. Waltham: UpToDate Inc.; 2020. Available from: https://covid19.bangkokhealth.com/wpcontent/uploads/2021/01/Uptodate Coronavirus-disease-2019-COVID-19-2.pdf.

5. Saez M, Tobias A, Varga D, Barceló MA. Effectiveness of the measures to flatten the epidemic curve of COVID-19. The case of Spain. Sci Total Environ. 2020; 727: 138761.

6. Pratt M, Varela AR, Salvo D, Kohl III HW, Ding D. Attacking the pandemic of physical inactivity: What is holding us back? Br J Sports Med. 2020; 54: 760-762.

7. Sonza A, da Cunha de Sá-Caputo D, Sartorio A, Tamini S, Seixas A, Sanudo B, et al. COVID-19 lockdown and the behavior change on physical exercise, pain and psychological well-being: An international multicentric study. Int J Environ Res Public Health. 2021; 18: 3810.

8. Deschasaux-Tanguy M, Druesne-Pecollo N, Esseddik Y, Szabo de Edelenyi F, Alles B, Andreeva VA, et al. Diet and physical activity during the COVID-19 lockdown period (March-May 2020): Results from the French NutriNet-Sante cohort study. MedRxiv. 2020. Doi: 10.1101/2020.06.04.20121855.

9. Alfawaz H, Amer OE, Aljumah AA, Aldisi DA, Enani MA, Aljohani NJ, et al. Effects of home quarantine during COVID-19 lockdown on physical activity and dietary habits of adults in Saudi Arabia. Sci Rep. 2021; 11: 5904.

10. Tison GH, Avram R, Kuhar P, Abreau S, Marcus GM, Pletcher MJ, et al. Worldwide effect of COVID-19 on physical activity: A descriptive study. Ann Intern Med. 2020; 173: 767-770.

11. Narici M, Vito GD, Franchi M, Paoli A, Moro T, Marcolin G, et al. Impact of sedentarism due to the COVID-19 home confinement on neuromuscular, cardiovascular and metabolic health: Physiological and pathophysiological implications and recommendations for physical and nutritional countermeasures. Eur J Sport Sci. 2021; 21: 614-635.

12. Fiok K, Karwowski W, Gutierrez E, Saeidi M, Aljuaid AM, Davahli MR, et al. A study of the effects of the COVID-19 pandemic on the experience of back pain reported on Twitter ${ }^{\circledast}$ in the United States: A natural language processing approach. Int J Environ Res Public Health. 2021; 18: 4543.

13. Laddu DR, Lavie CJ, Phillips SA, Arena R. Physical activity for immunity protection: Inoculating populations with healthy living medicine in preparation for the next pandemic. Prog Cardiovasc Dis. 2021; 64: 102-104.

14. Pietiläinen KH, Kaprio J, Borg P, Plasqui G, Yki-Järvinen H, Kujala UM, et al. Physical inactivity and obesity: A vicious circle. Obesity. 2008; 16: 409-414.

15. Siordia Jr JA. Epidemiology and clinical features of COVID-19: A review of current literature. J Clin Virol. 2020; 127: 104357.

16. Kohut ML, Sim YJ, Yu S, Yoon KJ, Loiacono CM. Chronic exercise reduces illness severity, decreases viral load, and results in greater anti-inflammatory effects than acute exercise during influenza infection. J Infect Dis. 2009; 200: 1434-1442.

17. Lowder T, Padgett DA, Woods JA. Moderate exercise protects mice from death due to influenza virus. Brain Behav Immun. 2005; 19: 377-380.

18. Warren KJ, Olson MM, Thompson NJ, Cahill ML, Wyatt TA, Yoon KJ, et al. Exercise improves host response to influenza viral infection in obese and non-obese mice through different mechanisms. PloS ONE. 2015; 10: e0129713.

19. Davis JM, Kohut ML, Colbert LH, Jackson DA, Ghaffar A, Mayer EP. Exercise, alveolar macrophage function, and susceptibility to respiratory infection. J Appl Physiol. 1997; 83: 1461-1466. 
20. Murphy EA, Davis JM, Carmichael MD, Gangemi JD, Ghaffar A, Mayer EP. Exercise stress increases susceptibility to influenza infection. Brain Behav Immun. 2008; 22: 1152-1155.

21. Nieman DC, Johanssen LM, Lee JW, Arabatzis K. Infectious episodes in runners before and after the Los Angeles marathon. J Sports Med Phys Fitness. 1990; 30: 316-328.

22. Nieman DC. Exercise, upper respiratory tract infection, and the immune system. Med Sci Sports Exerc. 1994; 26: 128-139.

23. Krüger K, Mooren FC, Pilat $\mathrm{C}$. The immunomodulatory effects of physical activity. Curr Pharm Des. 2016; 22: 3730-3748.

24. Simpson RJ, Campbell JP, Gleeson M, Krüger K, Nieman DC, Pyne DB, et al. Can exercise affect immune function to increase susceptibility to infection? Exerc Immunol Rev. 2020; 26: 8-22.

25. Hamer M, Kivimäki M, Gale CR, Batty GD. Lifestyle risk factors, inflammatory mechanisms, and COVID-19 hospitalization: A community-based cohort study of 387,109 adults in UK. Brain Behav Immun. 2020; 87: 184-187.

26. Tavakol Z, Ghannadi S, Tabesh MR, Halabchi F, Noormohammadpour P, Akbarpour S, et al. Relationship between physical activity, healthy lifestyle and COVID-19 disease severity; a crosssectional study. J Public Health. 2021. Doi: 10.1007/s10389-020-01468-9.

27. Brawner CA, Ehrman JK, Bole S, Kerrigan DJ, Parikh SS, Lewis BK, et al. Inverse relationship of maximal exercise capacity to hospitalization secondary to coronavirus disease 2019. Mayo Clin Proc. 2021; 96: 32-39.

28. McMURRAY RG, Ainsworth BE, Harrell JS, Griggs TR, Williams OD. Is physical activity or aerobic power more influential on reducing cardiovascular disease risk factors? Med Sci Sports Exerc. 1998; 30: 1521-1529.

29. Dwyer MJ, Pasini M, De Dominicis S, Righi E. Physical activity: Benefits and challenges during the COVID-19 pandemic. Scand J Med Sci Sports. 2020; 30: 1291-1294.

30. Guan WJ, Ni ZY, Hu Y, Liang WH, Ou CQ, He JX, et al. Clinical characteristics of coronavirus disease 2019 in China. N Engl J Med. 2020; 382: 1708-1720.

31. Livingston E, Bucher K. Coronavirus disease 2019 (COVID-19) in Italy. JAMA. 2020; 323: 1335.

32. Craig $C L$, Marshall $A L$, Sjöström $M$, Bauman $A E$, Booth $M L$, Ainsworth $B E$, et al. International physical activity questionnaire: 12-country reliability and validity. Med Sci Sports Exerc. 2003; 35: 1381-1395.

33. Lee PH, Macfarlane DJ, Lam TH, Stewart SM. Validity of the international physical activity questionnaire short form (IPAQ-SF): A systematic review. Int J Behav Nutr Phys Act. 2011; 8: 115.

34. US Department of Health and Human Services. Physical activity guidelines for Americans. Washington: US Department of Health and Human Services; 2018. Available from: https://health.gov/sites/default/files/2019-09/Physical Activity Guidelines 2nd edition.pdf.

35. Ammar A, Brach M, Trabelsi K, Chtourou H, Boukhris O, Masmoudi L, et al. Effects of COVID-19 home confinement on physical activity and eating behaviour Preliminary results of the ECLBCOVID19 international online-survey. MedRxiv. 2020. Doi: 10.1101/2020.05.04.20072447.

36. Nieman DC, Henson DA, Austin MD, Sha W. Upper respiratory tract infection is reduced in physically fit and active adults. Br J Sports Med. 2011; 45: 987-992.

37. Chubak J, McTiernan A, Sorensen B, Wener MH, Yasui Y, Velasquez M, et al. Moderate-intensity exercise reduces the incidence of colds among postmenopausal women. Am J Med. 2006; 119: 937-942.e5.

38. Halabchi F, Mazaheri R, Sabeti K, Yunesian M, Alizadeh Z, Ahmadinejad Z, et al. Regular sports 
participation as a potential predictor of better clinical outcome in adult patients with COVID-19: A large cross-sectional study. J Phys Act Health. 2020; 18: 8-12.

39. Sallis R, Young DR, Tartof SY, Sallis JF, Sall J, Li Q, et al. Physical inactivity is associated with a higher risk for severe COVID-19 outcomes: A study in 48440 adult patients. Br J Sports Med. 2021. Doi: $10.1136 /$ bjsports-2021-104080.

40. Nieman DC, Nehlsen-Cannarella SL, Markoff PA, Balk-Lamberton AJ, Yang H, Chritton DB, et al. The effects of moderate exercise training on natural killer cells and acute upper respiratory tract infections. Int J Sports Med. 1990; 11: 467-473.

41. Nieman DC. Exercise effects on systemic immunity. Immunol Cell Biol. 2000; 78: 496-501.

42. Petrilli CM, Jones SA, Yang J, Rajagopalan H, O'Donnell LF, Chernyak Y, et al. Factors associated with hospitalization and critical illness among 4,103 patients with COVID-19 disease in New York City. MedRxiv. 2020. Doi: 10.1101/2020.04.08.20057794.

43. Cai $Q$, Chen F, Wang T, Luo F, Liu X, Wu Q, et al. Obesity and COVID-19 severity in a designated hospital in Shenzhen, China. Diabetes Care. 2020; 43: 1392-1398.

44. Simonnet A, Chetboun M, Poissy J, Raverdy V, Noulette J, Duhamel A, et al. High prevalence of obesity in severe acute respiratory syndrome coronavirus-2 (SARS-CoV-2) requiring invasive mechanical ventilation. Obesity. 2020; 28: 1195-1199.

45. Halabchi F, Ahmadinejad Z, Selk-Ghaffari M. COVID-19 epidemic: exercise or not to exercise; that is the question. Asian J Sports Med. 2020; 11: e102630.

46. Melo-Oliveira ME, Sá-Caputo D, Bachur JA, Paineiras-Domingos LL, Sonza A, Lacerda AC, et al. Reported quality of life in countries with cases of COVID19: A systematic review. Expert Rev Respir Med. 2021; 15: 213-220.

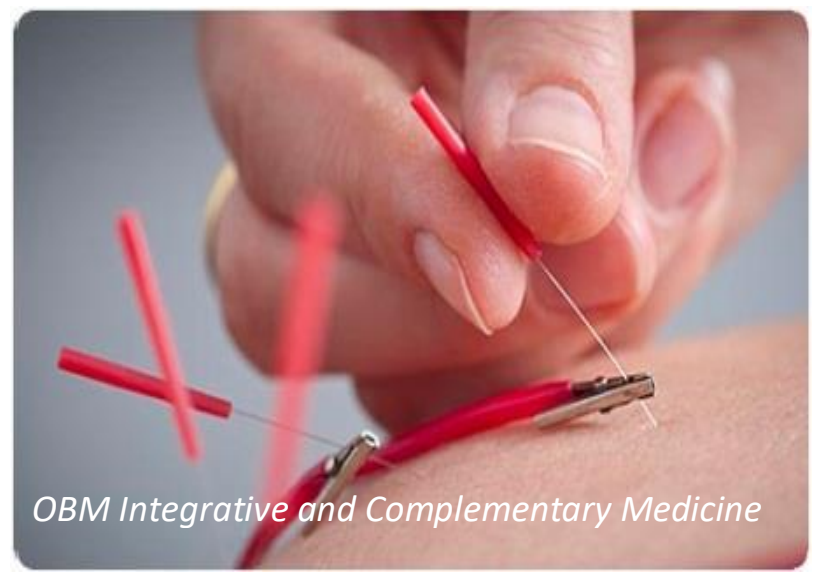

Enjoy OBM Integrative and Complementary Medicine by:

1. Submitting a manuscript

2. Joining in volunteer reviewer bank

3. Joining Editorial Board

4. Guest editing a special issue

For more details, please visit: http://www.lidsen.com/journals/icm 